$\begin{array}{lr}\text { Dept. OF Math./CMA } & \text { University of Oslo } \\ \text { PuRE Mathematics } & \text { No } 17 \\ \text { ISSN 0806-2439 } & \text { OCtober } 2007\end{array}$

\title{
Risk indifference pricing in jump diffusion markets
}

\author{
Bernt Øksendal ${ }^{1), 2)} \quad$ Agnès Sulem ${ }^{3)}$
}

Revised December 18, 2008

\begin{abstract}
We study the risk indifference pricing principle in incomplete markets: The (seller's) risk indifference price $p_{\text {risk }}^{\text {seller }}$ is the initial payment that makes the risk involved for the seller of a contract equal to the risk involved if the contract is not sold, with no initial payment. We use stochastic control theory and PDE methods to find a formula for $p_{\text {risk }}^{\text {seller }}$ and similarly for $p_{\text {risk }}^{\text {buyer }}$. In particular, we prove that

$$
p_{\text {low }} \leq p_{\text {risk }}^{\text {buyer }} \leq p_{\text {risk }}^{\text {seller }} \leq p_{\text {up }}
$$
\end{abstract}

where $p_{\text {low }}$ and $p_{\text {up }}$ are the lower and upper hedging prices, respectively.

\section{Introduction}

Consider a financial market with two investment possibilities

(i) a risk free investment, with unit price $S_{0}(t)=1$ at all times $t \geq 0$.

1) Center of Mathematics for Applications (CMA), University of Oslo, Box 1053 Blindern, N-0316 Oslo, Norway, Email: oksendal@math.uio.no

2) Norwegian School of Economics and Business Administration, Helleveien 30, N-5045 Bergen, Norway

3) INRIA Paris-Rocquencourt, Domaine de Voluceau, Rocquencourt, B.P. 105, F78153 Le Chesnay Cedex, France, Email: agnes.sulem@inria.fr 
(ii) a risky investment, where the unit price is described by a semimartingale $S(t)$ on a filtered probability space $\left(\Omega, \mathcal{F},\left\{\mathcal{F}_{t}\right\}_{t \geq 0} P\right)$.

A contingent claim with maturity $T>0$ (also called a $T$-claim) is an $\mathcal{F}_{T}$-measurable random variable $G=G(\omega) ; \omega \in \Omega$, representing the payoff that the seller of a contract guarantees to deliver to the buyer at time $T$.

A portfolio in this market is an $\mathcal{F}_{t}$-predictable process $\pi(t)=\pi(t, \omega)$, representing the number of units of the risky asset held at time $t$, which is self-financing, i.e. satisfies the equation

$$
X_{x}^{(\pi)}(t):=\pi(t) S(t)=x+\int_{0}^{t} \pi(s) d S(s)
$$

The process $X_{x}^{(\pi)}(t)$ is called the wealth process associated to the portfolio $\pi$ and with initial value $X_{x}^{(\pi)}(0)=x$.

The market is called complete if for every bounded $T$-claim $G$ there exists $x \in \mathbb{R}$ and a portfolio $\pi$ such that

$$
X_{x}^{(\pi)}(T)=G \quad \text { a.s. }
$$

If this is the case, then there is a unique linear arbitrage free pricing rule at time $t=0$ for a contract with payoff $G$ at time $t=T$. This price is

$$
p(G)=E_{Q}[G]
$$

where $E_{Q}$ denotes expectation with respect to the (unique) equivalent martingale measure (EMM).

In incomplete markets, however, the situation is not so clear. There are infinitely many equivalent martingale measures $Q$ and it is not clear which one to use in the pricing formula (1.3). In this paper we study a class of incomplete markets, namely the jump diffusion markets, and we investigate a pricing formula based on the risk indifference principle. This gives a price $p_{\text {risk }}^{\text {seller }}$ for the seller and a corresponding price $p_{\text {risk }}^{\text {buyer }}$ for the buyer of the contract. We prove that we always have

$$
p_{\text {low }} \leq p_{\text {risk }}^{\text {buyer }} \leq p_{\text {risk }}^{\text {seller }} \leq p_{\text {up }}
$$

where $p_{\text {low }}$ and $p_{\text {up }}$ are the lower and upper hedging prices, respectively. (See Section 2 for details). Thus the gap between the seller and buyer prices is 
smaller with the risk indifference pricing than with upper and lower hedging pricing.

Our paper is organized as follows: In Section 2 we give a short presentation of some principles of pricing in incomplete markets. In Section 3 we give a precise formulation of our jump diffusion market model. In Section 4 we use dynamic programming for stochastic differential games to find an explicit relation between the value function $\Phi_{G}$ of the stochastic differential game involved in the risk indifferent pricing and the value function $\Psi_{G}$ for a corresponding stochastic control problem involving only equivalent martingale measures. In Section 5 we prove the same relation in the setting of viscosity solutions of the corresponding HJBI and HJB equations. Finally, in Section 6 we apply the results from the earlier sections to derive formulas for the risk indifference prices $p_{\text {risk }}^{\text {seller }}$ and $p_{\text {risk }}^{\text {buyer }}$ and we discuss conditions under which these prices coincide.

\section{Pricing in incomplete markets}

Superreplication. The upper hedging price of $G$ at time $t=0$ is defined by

$$
p_{\text {up }}(G)=\inf \left\{x ; \text { there exists } \pi \in \mathcal{P} \text { such that } X_{x}^{(\pi)}(T) \geq G \text { a.s. }\right\}
$$

where $\mathcal{P}$ denotes the set of admissible portfolios. This price is sometimes called the seller's price, because it represents the minimal initial payment $x$ needed in order to be able to hedge a terminal wealth $X_{x}^{(\pi)}(T)$ which is no less than the guaranteed payoff $G$, a.s. One can show that

$$
p_{\text {up }}(G)=\sup _{Q \in \mathcal{M}_{1}} E_{Q}[G]
$$

(see e.g. $[\mathrm{Ku}]$ ) where $\mathcal{M}_{1}$ denotes the set of equivalent martingale measures $Q$, i.e. the set of probability measures $Q$ on $\mathcal{F}_{T}$ such that $Q \ll P$ and $P \ll Q$ and the discounted price process $\mathcal{S}(t)$ is a martingale with respected to $Q$.

Similarly, the lower hedging price (or the buyer's price) can be defined by

$$
p_{\text {low }}(G)=\inf _{Q \in \mathcal{M}_{1}} E_{Q}[G]
$$

In incomplete markets there are infinitely many measures $Q \in \mathcal{M}_{1}$ and there is usually a big gap between $p_{\text {low }}(G)$ and $p_{\text {up }}(G)$. 
Utility indifference pricing. This pricing principle was introduced by Hodges and Neuberger $[\mathrm{HN}]$. It is based on a given (chosen) utility function $U: \mathbb{R} \rightarrow \mathbb{R} \cup\{-\infty\}$

(i) If a person sells a liability to pay out the amount $G(\omega)$ at time $T$ and receives an initial payment $p$ for such a contract, the maximal expected utility for the seller is

$$
V_{G}(x+p)=\sup _{\pi \in \mathcal{P}} E\left[U\left(X_{x+p}^{(\pi)}(T)-G\right)\right]
$$

where $x$ is the sellers wealth before the contract is being made.

(ii) If, on the other hand, no such contract is made, the maximal expected utility is

$$
V_{0}(x)=\sup _{\pi \in \mathcal{P}} E\left[U\left(X_{x}^{(\pi)}(T)\right)\right]
$$

The (seller's) utility indifference price is the value of the initial payment $p$ that makes the seller indifferent to whether to sell the contract or not, i.e. $p$ is the solution $p=p_{\text {utility }}$ of the equation

$$
V_{G}(x+p)=V_{0}(x)
$$

To find $p$ one has to solve the two stochastic control problems (2.4) and (2.5). In general this is difficult. (See e.g. [MZ], [ST], [BeM]). In the case of exponential utility functions, the utility indifference price is independent of the initial wealth.

Risk indifference pricing. The purpose of this paper is to study a pricing principle based on risk rather than utility. Thus the starting point is a given convex risk measure

$$
\rho: \mathbb{F} \rightarrow \mathbb{R}
$$

where $\mathbb{F}$ is the set of $\mathcal{F}_{T}$-measurable random variables (see below for a definition). We may regard $\mathbb{F}$ as the family of all possible financial positions at time $T$, and if $F \in \mathbb{F}$ then $\rho(F)$ may be interpreted as the the amount the agent has to hold to cancel the risk associated with his risky position $F$, that is $\rho(F+\rho(F))=0$ (see $[\mathrm{BE}])$. We now argue as in the utility indifference case: 
(i) If a person sells a contract which guarantees a payoff $G \in \mathbb{F}$ at time $T$ and receives an initial payment $p$ for this, then the minimal risk involved for the seller is

$$
\Phi_{G}(x+p)=\inf _{\pi \in \mathcal{P}} \rho\left(X_{x+p}^{(\pi)}(T)-G\right)
$$

(ii) If, on the other hand, no contract is sold (and hence no initial payment is received), then the minimal risk for the person is

$$
\Phi_{0}(x)=\inf _{\pi \in \mathcal{P}} \rho\left(X_{x}^{(\pi)}(T)\right) .
$$

Definition 2.1 The(seller's) risk indifference price $p=p_{\text {risk }}$ of the claim $G \in \mathbb{F}$ is the solution $p$ of the equation

$$
\Phi_{G}(x+p)=\Phi_{0}(x)
$$

Thus $p_{\text {risk }}$ is the initial payment that makes a person risk indifferent between selling the contract with liability payoff $G$ and not selling the contract (and not receiving any payment either).

A convex risk measure $\rho$ is usually defined as a map $\rho: \mathbb{F} \rightarrow \mathbb{R}$ satisfying certain axioms (convexity, monotonicity and translation invariance). (See $[\mathrm{ADEH}],[\mathrm{FR}]$ or $[\mathrm{FS}]$.) Note that because of the translation property of risk measures the risk indifference price $p$ is always independent on the initial wealth $x$.

We will use the following representation of convex risk measures:

Theorem $2.2([\mathbf{F S}],[\mathbf{F R}]) A$ map $\rho: \mathbb{F} \rightarrow \mathbb{R}$ is a convex risk measure if and only if there exists a family $\mathcal{L}$ of probability measures $Q \ll P$ on $\mathcal{F}_{T}$ and a convex "penalty function" $\zeta: \mathcal{L} \rightarrow \mathbb{R} \cup\{+\infty\}$ with $\inf _{Q \in \mathcal{L}} \zeta(Q)=0$ such that

$$
\rho(F)=\sup _{Q \in \mathcal{L}}\left\{E_{Q}[-F]-\zeta(Q)\right\} ; \quad F \in \mathbb{F} .
$$

In view of this representation we see that choosing a risk measure $\rho$ is equivalent to choosing the family $\mathcal{L}$ of measures and the penalty function $\zeta$. If we choose $\zeta=0$ then $\rho$ becomes a coherent risk measure (see $[\mathrm{ADEH}]$ and [D]). 
Using the representation (2.10) we see that the problem of finding the risk indifference price $p=p_{\text {risk }}$ given by (2.9) amounts to solving the following two stochastic differential (zero-sum) game problems:

$$
\Phi_{G}(x+p)=\inf _{\pi \in \mathcal{P}}\left(\sup _{Q \in \mathcal{L}}\left\{E_{Q}\left[-X_{x+p}^{(\pi)}(T)+G\right]-\zeta(Q)\right\}\right)
$$

and

$$
\Phi_{0}(x)=\inf _{\pi \in \mathcal{P}}\left(\sup _{Q \in \mathcal{L}}\left\{E_{Q}\left[-X_{x}^{(\pi)}(T)\right]-\zeta(Q)\right\}\right),
$$

for a given family of measures $\mathcal{L}$ and a given penalty function $\zeta$.

We will make a choice of $\mathcal{L}$ which makes it possible to solve such games using Hamilton-Jacobi-Bellman-Isaacs (HJBI) equations.

The idea of using a risk indifference principle rather than a utility indifference principle has appeared in various settings in several papers recently. See in particular $[\mathrm{X}]$ and $[\mathrm{KS}]$ with the references therein. However, the methods and results of these papers are different from ours. Perhaps the paper which is closest to ours is $[\mathrm{X}]$, where the risk measure pricing is studied. The pricing principle of $[\mathrm{X}]$ is essentially the same as ours. However, $[\mathrm{X}]$ does not study the corresponding stochastic differential game problem and does not relate it explicitly to the upper and lower hedging problem as we do in Theorems 4.2 and 5.2. In particular, $[\mathrm{X}]$ does not obtain the identities (6.3) and (6.5).

\section{Precise formulation of the model}

Let $\eta(t)=\eta(t, \omega) ;(t, \omega) \in[0, \infty) \times \Omega$ be a Lévy process on a filtered probability space $\left(\Omega ; \mathcal{F},\left\{\mathcal{F}_{t}\right\}_{t \geq 0}, P\right)$. For simplicity we assume that

$$
E\left[\eta^{2}(t)\right]<\infty \quad \text { for all } t \geq 0 .
$$

Then by the Itô-Lévy decomposition theorem we can write

$$
\eta(t)=a t+b B(t)+\int_{0}^{t} \int_{\mathbb{R}_{0}} z \tilde{N}(d s, d z) ; \quad t \geq 0 \quad\left(\mathbb{R}_{0}=\mathbb{R} \backslash\{0\}\right)
$$

where $a$ and $b$ are constants, $B(t)$ is a Brownian motion and

$$
\tilde{N}(d t, d z)=N(d t, d z)-\nu(d z) d t
$$


is the compensated jump measure of $\eta, \nu$ being the Lévy measure and $N$ the jump measure, respectively.

Because of our assumption (3.1) we have that

$$
\int_{\mathbb{R}_{0}} z^{2} \nu(d z)<\infty
$$

We refer the reader to [Ap], [B] and [Sa] for more information about Lévy processes. A short review useful for this paper is given in [ØS].

Consider a financial market where there are two investment possibilities:

(i) A risk free investment, with discounted unit price $S_{0}(t)=1 ; t \in[0, T]$ where $T>0$ is a fixed terminal time.

(ii) A risky investment, where the discounted unit price $S(t)$ at time $t$ is given by

$$
\begin{aligned}
& d S(t)=S\left(t^{-}\right)[\alpha(t) d t+\beta(t) d B(t) \\
& \left.\quad+\int_{\mathbb{R}_{0}} \gamma(t, z) \tilde{N}(d t, d z)\right] \quad S(0)=s>0 ; \quad t \in[0, T]
\end{aligned}
$$

Here $\alpha(t), \beta(t)$ and $\gamma(t, z)$ are $\mathcal{F}_{t}$-predictable processes. We assume that $\gamma(t, z)>-1$ for a.a. $t, z$ and that

$$
\int_{0}^{T}\left\{|\alpha(t)|+\beta^{2}(t)+\int_{\mathbb{R}_{0}}|\log (1+\gamma(t, z))|^{2} \nu(d z)\right\} d t<\infty \quad \text { a.s. }
$$

We represent a portfolio in this market by the number $\pi(t)$ of units of the risky asset held at time $t$. The dynamics of the corresponding discounted wealth $X(t)=X^{(\pi)}(t)$ will then be

$$
\begin{aligned}
& d X(t)=\pi(t) d S(t)=\pi(t) S\left(t^{-}\right)[\alpha(t) d t+\beta(t) d B(t) \\
& \left.+\int_{\mathbb{R}_{0}} \gamma(t, z) \tilde{N}(d t, d z)\right] ; \quad t \in[0, T] \\
& X(0)=x>0
\end{aligned}
$$


The portfolio $\pi(t)$ is called admissible if $\pi(t)$ is predictable and satisfies

$$
\begin{aligned}
& \int_{0}^{T}\left\{|\alpha(t)||\pi(t)| S(t)+\beta^{2}(t) \pi^{2}(t) S^{2}(t)\right. \\
& \left.\quad+\pi^{2}(t) S^{2}(t) \int_{\mathbb{R}_{0}} \gamma^{2}(t, z) \nu(d z)\right\} d t<\infty \quad \text { a.s. }
\end{aligned}
$$

and

$$
X^{(\pi)}(t) \geq 0 \quad \text { for } t \in[0, T] \text {, a.s. }
$$

The set of all admissible portfolios is denoted by $\mathcal{P}$.

It is well-known that such a market is in general incomplete. Therefore there is no unique equivalent martingale measure and hence no unique method of pricing a given contingent claim with discounted payoff $G$ in an arbitrage free way.

We first describe two sets $\mathcal{L}, \mathcal{M}$ of measures. For given $\mathcal{F}_{t}$-predictable processes $\theta_{0}(t)$ and $\theta_{1}(t, z) ; t \geq 0, z \in \mathbb{R}_{0}$ such that

$$
\int_{0}^{T}\left\{\theta_{0}^{2}(t)+\int_{\mathbb{R}_{0}}\left(\log \left(1+\theta_{1}(t, z)\right)\right)^{2} \nu(d z)\right\} d t<\infty \text { a.s. }
$$

(in particular, $\theta_{1}(t, z) \geq-1$ for a.a. $\left.t, z, \omega\right)$, define the process $K_{\theta}(t)$ as the solution of the stochastic differential equation

$$
\begin{aligned}
d K_{\theta}(t) & =K_{\theta}\left(t^{-}\right)\left[\theta_{0}(t) d B(t)+\int_{\mathbb{R}_{0}} \theta_{1}(t, z) \tilde{N}(d t, d z)\right] ; \quad t \in[0, T] \\
K_{\theta}(0) & =k>0
\end{aligned}
$$

i.e.

$$
\begin{gathered}
K_{\theta}(t)=k \exp \left(\int_{0}^{t} \theta_{0}(s) d B(s)-\frac{1}{2} \int_{0}^{t} \theta_{0}^{2}(s) d s\right. \\
+\int_{0}^{t} \int_{\mathbb{R}_{0}} \log \left(1+\theta_{1}(s, \theta)\right) \tilde{N}(d s, d z)
\end{gathered}
$$




$$
\left.+\int_{0}^{t} \int_{\mathbb{R}_{0}} \log \left(1+\theta_{1}(s, z)\right)-\theta_{1}(s, z) \nu(d z) d s\right) ; \quad t \in[0, T] .
$$

Then define the measure $Q_{\theta}$ by

$$
d Q_{\theta}(\omega)=K_{\theta}(T) d P(\omega) \quad \text { on } \mathcal{F}_{T}
$$

To put our problems (2.11) and (2.12) into a Markovian framework we define our (controlled) process $Y(t)=Y^{\theta, \pi}(t) \in \mathbb{R}^{3}$, as follows:

$$
\begin{aligned}
d Y(t)= & {\left[\begin{array}{l}
d Y_{1}(t) \\
d Y_{2}(t) \\
d Y_{3}(t)
\end{array}\right]=\left[\begin{array}{c}
d K_{\theta}(t) \\
d S(t) \\
d X^{(\pi)}(t)
\end{array}\right]=\left[\begin{array}{l}
0 \\
S\left(t^{-}\right) \alpha(t) \\
\left.S\left(t^{-}\right) \alpha(t) \pi(t)\right]
\end{array}\right] d t } \\
& +\left[\begin{array}{c}
K_{\theta}\left(t^{-}\right) \theta_{0}(t) \\
S\left(t^{-}\right) \beta(t) \\
S\left(t^{-}\right) \beta(t) \pi(t)
\end{array}\right] d B(t)+\int\left[\begin{array}{c}
K_{\theta}\left(t^{-}\right) \theta_{1}(t, z) \\
S\left(t^{-}\right) \gamma(t, z) \\
S\left(t^{-}\right) \pi(t) \gamma(t, z)
\end{array}\right] \tilde{N}(d t, d z)
\end{aligned}
$$

and

$$
Y(0)=y=\left(y_{1}, y_{2}, y_{3}\right)=(k, s, x) \in \mathbb{R}^{3} .
$$

Similarly we let $\tilde{Y}(t)=Y^{\theta}(t)$ be the state process obtained by deleting the 3rd component, $X^{(\pi)}(T)$, from $Y(t)$, i.e.

$$
\tilde{Y}(t)=\left[\begin{array}{c}
Y_{1}(t) \\
Y_{2}(t)
\end{array}\right]=\left[\begin{array}{c}
K_{\theta}(t) \\
S(t)
\end{array}\right] ; \quad \tilde{Y}(0)=\tilde{y}=\left(y_{1}, y_{2}\right)=(k, s) \in \mathbb{R}^{2}
$$

We assume that all the coefficients are Markovian with respect to $\tilde{Y}(\cdot)$, i.e.

$$
\alpha(t)=\bar{\alpha}(t, \tilde{Y}(t)), \quad \beta(t)=\bar{\beta}(t, \tilde{Y}(t)) \quad \text { and } \gamma(t)=\bar{\gamma}(t, \tilde{Y}(t), z),
$$

for given functions

$$
\bar{\alpha}: \mathbb{R}^{3} \rightarrow \mathbb{R}, \quad \bar{\beta}: \mathbb{R}^{3} \rightarrow \mathbb{R} \quad \text { and } \quad \bar{\gamma}: \mathbb{R}^{3} \times \mathbb{R}_{0} \rightarrow \mathbb{R}
$$

For simplicity of notation we will in the following not distinguish between $\alpha$ and $\bar{\alpha}$ etc., i.e. we write (with abuse of notation)

$$
\alpha(t)=\alpha(t, \tilde{Y}(t)) \quad \text { etc. }
$$


Let $\mathbb{L}$ be the set of all $\tilde{Y}(t)$-Markovian controls $\theta(t)=\left(\theta_{0}(t), \theta_{1}(t, z)\right)$, where $\theta_{0}(t)=\theta_{0}(t, \tilde{Y}(t))$ and $\theta_{1}(t, z)=\theta_{1}(t, \tilde{Y}(t), z), z \in \mathbb{R}$, satisfying (3.11) and such that

$$
E\left[K_{\theta}(T)\right]=K_{\theta}(0)=k>0
$$

(this implies that $K_{\theta}(t)$ is a martingale).

Similarly let

$$
\Pi=\{\pi \in \mathcal{P} ; \pi \text { is } Y(t) \text {-Markovian, i.e. } \pi(t)=\pi(t, Y(t))\}
$$

We now define two sets $\mathcal{L}, \mathcal{M}$ of measures as follows:

$$
\begin{aligned}
\mathcal{L} & =\left\{Q_{\theta} ; \theta \in \mathbb{L}\right\}, \\
\mathcal{M} & =\left\{Q_{\theta} ; \theta \in \mathbb{M}\right\},
\end{aligned}
$$

where

$$
\mathbb{M}=\{\theta \in \mathbb{L} ; M \theta(t, \tilde{y})=0 \text { for all } t, \tilde{y}\}
$$

with

$$
\begin{aligned}
M \theta(t, \tilde{y})= & M(\theta)(t, k, s)=\alpha(t, \tilde{y})+\theta_{0}(t, \tilde{y}) \beta(t, \tilde{y}) \\
& +\int_{\mathbb{R}_{0}} \theta_{1}(t, \tilde{y}, z) \gamma(t, \tilde{y}, z) \nu(d z) ; \quad(t, \tilde{y}) \in[0, T] \times \mathbb{R}^{2} .
\end{aligned}
$$

Note that, by the Girsanov theorem, all the measures $Q_{\theta} \in \mathcal{M}$ with $K_{\theta}(0)=1$ are equivalent martingale measures. (See e.g. [ØS], Section 1.4.)

We now return to the stochastic differential game problems (2.11) and (2.12). We will assume that the penalty function $\zeta$ has the form

$$
\zeta\left(Q_{\theta}\right)=E\left[\int_{0}^{T} \int_{\mathbb{R}_{0}} \lambda\left(t, \theta_{0}(t, \tilde{Y}(t)), \theta_{1}(t, \tilde{Y}(t), z), \tilde{Y}(t), z\right) \nu(d z) d t+h(\tilde{Y}(T))\right]
$$

for some convex functions $\lambda \in C^{1}\left(\mathbb{R}^{4} \times \mathbb{R}_{0}\right), h \in C^{1}(\mathbb{R})$ s.t.

$$
E\left[\int_{0}^{T} \int_{\mathbb{R}_{0}}\left|\lambda\left(t, \theta_{0}(t, \tilde{Y}(t)), \theta_{1}(t, \tilde{Y}(t), z), \tilde{Y}(t), z\right)\right| \nu(d z) d t+|h(\tilde{Y}(T))|\right]<\infty
$$


for all $(\theta, \pi) \in \mathbb{L} \times \Pi$.

Moreover, we assume that the given claim $G$ has the (Markovian) form

$$
G=g(S(T))
$$

for some $g: \mathbb{R} \rightarrow \mathbb{R}$ such that

$$
E_{Q_{\theta}}[|g(S(T))|]<\infty \text { for all } \theta \in \mathbb{L}
$$

Using the $Y(t)$-notation, we see that problem (2.11) can be written as follows:

Problem A Find $\Phi_{G}(t, y)$ and $\left(\theta^{*}, \pi^{*}\right) \in \mathbb{L} \times \Pi$ (called an optimal pair) such that

$$
\Phi_{G}(t, y):=\inf _{\pi \in \Pi}\left(\sup _{\theta \in \mathbb{L}} J^{\theta, \pi}(t, y)\right)=J^{\theta^{*}, \pi^{*}}(t, y)
$$

where

$$
\begin{gathered}
J^{\theta, \pi}(t, y)=E^{t, y}\left[-\int_{t}^{T} \Lambda(\theta(u, \tilde{Y}(u))) d u-h(\tilde{Y}(T))\right. \\
\left.+K_{\theta}(T) g(S(T))-K_{\theta}(T) X^{(\pi)}(T)\right]
\end{gathered}
$$

and

$$
\Lambda(\theta)=\Lambda(\theta(t, \tilde{y}))=\int_{\mathbb{R}} \lambda\left(t, \theta_{0}(t, \tilde{y}), \theta_{1}(t, \tilde{y}, z), \tilde{y}, z\right) \nu(d z), \quad \tilde{y}=(k, s) .
$$

We will relate Problem A to the following stochastic control problem:

$$
\Psi_{G}=\sup _{Q \in \mathcal{M}}\left\{E_{Q}[G]-\zeta(Q)\right\}
$$

Putting this into a Markovian context as above, the problem gets the following form: 
Problem B Find $\Psi_{G}(t, \tilde{y})$ and $\check{\theta} \in \mathbb{M}$ such that

$$
\Psi_{G}(t, \tilde{y})=\sup _{\theta \in \mathbb{M}} J_{0}^{\theta}(t, \tilde{y})=J_{0}^{\check{\theta}}(t, \tilde{y})
$$

where

$$
\begin{gathered}
J_{0}^{\theta}(t, \tilde{y})=E^{t, \tilde{y}}\left[-\int_{t}^{T} \Lambda(\theta(u, \tilde{Y}(u))) d u-h(\tilde{Y}(T))\right. \\
\left.+K_{\theta}(T) g(S(T))\right] ; \quad \tilde{y}=(k, s) .
\end{gathered}
$$

Note that

$$
J^{\theta, \pi}(t, y)=J_{0}^{\theta}(t, \tilde{y})-E^{y}\left[K_{\theta}(T) X^{(\pi)}(T)\right]
$$

For given $(\theta, \pi) \in \mathbb{L} \times \Pi$ the process $Y^{\theta, \pi}(t)$ is Markovian with generator $A^{\theta, \pi}$ given by

$$
\begin{aligned}
& A^{\theta, \pi} \varphi(t, y)=\frac{\partial \varphi}{\partial t}+\alpha s \frac{\partial \varphi}{\partial s}+s \alpha \pi \frac{\partial \varphi}{\partial x} \\
& \quad+\frac{1}{2} \theta_{0}^{2} k^{2} \frac{\partial^{2} \varphi}{\partial k^{2}}+\frac{1}{2} \beta^{2} s^{2} \frac{\partial^{2} \varphi}{\partial s^{2}}+\frac{1}{2} s^{2} \beta^{2} \pi^{2} \frac{\partial^{2} \varphi}{\partial x^{2}} \\
& \quad+\theta_{0} \beta k s \frac{\partial^{2} \varphi}{\partial k \partial s}+\theta_{0} \pi \beta k s \frac{\partial^{2} \varphi}{\partial k \partial x}+\pi \beta^{2} s^{2} \frac{\partial^{2} \varphi}{\partial s \partial x} \\
& \quad+\int_{\mathbb{R}_{0}}\left\{\varphi\left(t, k+k \theta_{1}, s+s \gamma, x+s \pi \gamma\right)\right. \\
& \left.\quad-\varphi(t, k, s, x)-k \theta_{1} \frac{\partial \varphi}{\partial k}-s \gamma \frac{\partial \varphi}{\partial s}-s \pi \gamma \frac{\partial \varphi}{\partial x}\right\} \nu(d z), \\
& \text { for all } \varphi=\varphi(t, k, s, x) \in C^{1,2}\left([0, T] \times \mathbb{R}_{+}^{3}\right) .
\end{aligned}
$$

(See e.g. $[\varnothing \mathrm{S}]$ for more information about stochastic control of jump diffusions.)

If we delete the third component $Y_{3}(t)=X^{(\pi)}(t)$ and only consider the corresponding Markov process $\tilde{Y}^{\theta}(t)=\left(K_{\theta}(t), S(t)\right)$, its generator $A^{\theta}$ is given by

$$
\begin{aligned}
& A^{\theta} \psi(t, \tilde{y})=\frac{\partial \psi}{\partial t}+\alpha s \frac{\partial \psi}{\partial s} \\
& \quad+\frac{1}{2} \theta_{0}^{2} k^{2} \frac{\partial^{2} \psi}{\partial k^{2}}+\frac{1}{2} \beta^{2} s^{2} \frac{\partial^{2} \psi}{\partial s^{2}}+\theta_{0} \beta k s \frac{\partial^{2} \psi}{\partial k \partial s}
\end{aligned}
$$




$$
\begin{aligned}
& +\int_{\mathbb{R}_{0}}\left\{\psi\left(t, k+k \theta_{1}, s+s \gamma\right)-\psi(t, k, s)\right. \\
& \left.-k \theta_{1} \frac{\partial \psi}{\partial k}-s \gamma \frac{\partial \psi}{\partial s}\right\} \nu(d z), \\
& \text { for all } \left.\psi=\psi(t, k, s) \in C^{1,2}([0, T]) \times \mathbb{R}_{+}^{2}\right) .
\end{aligned}
$$

The following simple result will be useful:

Lemma 3.1 Let $\psi \in C^{1,2}\left([0, T] \times \mathbb{R}_{+}^{2}\right)$ and define

$$
\varphi(t, k, s, x):=\psi(t, k, s)-k x .
$$

Then, with $\tilde{y}=\left(y_{1}, y_{2}\right)=(k, s)$ as before,

$$
\begin{aligned}
A^{\theta, \pi} \varphi(t, y)= & A^{\theta} \psi(t, \tilde{y})-k s \pi(y)\left[\alpha(t, \tilde{y})+\theta_{0}(t, \tilde{y}) \beta(t, \tilde{y})\right. \\
& \left.+\int_{\mathbb{R}_{0}} \theta_{1}(t, \tilde{y}, z) \gamma(t, \tilde{y}, z) \nu(d z)\right]
\end{aligned}
$$

Proof. From (3.32) and (3.33) we see that

$$
A^{\theta, \pi} \psi(t, \tilde{y})=A^{\theta} \psi(t, \tilde{y}),
$$

so it only remains to compute

$$
\begin{aligned}
& A^{\theta, \pi}(k x)=s \alpha \pi k+s \theta_{0} \pi \beta k+\int_{\mathbb{R}_{0}}\left\{\left(k+k \theta_{1}\right)(x+s x \pi \gamma)\right. \\
& \left.\quad-k x-k \theta_{1} x-s \pi \gamma k\right\} \nu(d z)=s k \pi\left[\alpha+\theta_{0} \beta+\int_{\mathbb{R}_{0}} \theta_{1} \gamma \nu(d z)\right] .
\end{aligned}
$$

Lemma 3.2 Let $\psi$ and $\varphi$ be as in Lemma 3.1. We put $\Theta=\left\{\left(\theta_{0}, \theta_{1}\right) ; \theta_{0} \in \mathbb{R}\right.$ and $\theta_{1}$ is a function from $\mathbb{R}_{0}$ to $\left.\mathbb{R}\right\}$. Suppose that for all $\pi \in \mathbb{R},(t, k, x) \in \tilde{\mathcal{S}}$ there exists a maximum point $\hat{\theta}=\hat{\theta}(\pi)$ of the function

$$
\theta \rightarrow A^{\theta} \psi-\Lambda \theta-k s \pi M \theta ; \quad \theta \in \Theta
$$

and that $\pi \rightarrow \hat{\theta}(\pi)$ is a $C^{1}$-function. Moreover, suppose the map

$$
\pi \rightarrow A^{\hat{\theta}(\pi)} \psi-\Lambda \hat{\theta}(\pi)-k s \pi M \hat{\theta}(\pi) ; \quad \pi \in \mathbb{R}
$$


has a minimum point $\hat{\pi} \in \mathbb{R}$. Define

$$
\check{\theta}:=\hat{\theta}(\hat{\pi}) .
$$

Then

$$
M \check{\theta}=0
$$

and

$$
\inf _{\pi}\left(\sup _{\theta}\left\{A^{\theta, \pi} \varphi-\Lambda \theta\right\}\right)=A^{\check{\theta}} \psi-\Lambda \check{\theta}=\sup _{\theta: M \theta=0}\left\{A^{\theta} \psi-\Lambda \theta\right\}
$$

Proof. The first order conditions for a maximum point $\hat{\theta}=\hat{\theta}(\pi)$ of the map

$$
\theta \rightarrow A^{\theta} \psi-\Lambda \theta-k s \pi M \theta ; \quad \theta \in \Theta
$$

(for fixed $t, k, s$ and $\pi$ ) are

$$
\nabla_{\theta}\left(A^{\theta} \psi-\Lambda \theta-k s \pi M \theta\right)_{\theta=\hat{\theta}}=0,
$$

where $\nabla_{\theta}=\left(\frac{\partial}{\partial \theta_{0}}, \frac{\partial}{\partial \theta_{1}}\right)$ denotes the gradient operator. The first order condition for a minimum point $\hat{\pi}$ of the map

$$
\pi \rightarrow A^{\hat{\theta}(\pi)} \psi-\Lambda \hat{\theta}(\pi)-k s \pi M \hat{\theta}(\pi) ; \quad \pi \in \mathbb{R}
$$

is, by the chain rule,

$$
\nabla_{\theta}\left(A^{\theta} \psi-\Lambda \theta-k s \pi M \theta\right)_{\theta=\hat{\theta}(\hat{\pi})}\left(\frac{d \hat{\theta}(\pi)}{d \pi}\right)_{\pi=\hat{\pi}}-k s M \hat{\theta}(\hat{\pi})=0 .
$$

By (3.39) the first term is 0 and we conclude that

$$
M \hat{\theta}(\hat{\pi})=0
$$

Therefore

$$
\check{\theta}:=\hat{\theta}(\hat{\pi})
$$

satisfies the constraint $M \check{\theta}=0$, as claimed. Hence

$$
\begin{aligned}
& \inf _{\pi}\left(\sup _{\theta}\left\{A^{\theta} \psi-\Lambda \theta-k s \pi M \theta\right\}\right) \\
& \quad=\inf _{\pi}\left(A^{\hat{\theta}(\pi)} \psi-\Lambda \hat{\theta}(\pi)-k s \pi M \hat{\theta}(\pi)\right)
\end{aligned}
$$




$$
=A^{\check{\theta}} \psi-\Lambda \check{\theta} \leq \sup _{\theta: M \theta=0}\left\{A^{\theta} \psi-\Lambda \theta\right\} .
$$

On the other hand, we always have

$$
\begin{aligned}
& \inf _{\pi}\left(\sup _{\theta}\left\{A^{\theta} \psi-\Lambda \theta-k s \pi M \theta\right\}\right) \\
& \quad \geq \inf _{\pi}\left(\sup _{\theta: M \theta=0}\left\{A^{\theta} \psi-\Lambda \theta-k s \pi M \theta\right\}\right)=\sup _{\theta: M \theta=0}\left\{A^{\theta} \psi-\Lambda \theta\right\} .
\end{aligned}
$$

Combining (3.40) and (3.41) we get

$$
\begin{aligned}
\inf _{\pi}\left(\sup _{\theta}\left\{A^{\theta} \psi-\Lambda \theta-k s \pi M \theta\right\}\right) \\
\quad=A^{\check{\theta}} \psi-\Lambda \check{\theta}=\sup _{\theta: M \theta=0}\left\{A^{\theta} \psi-\Lambda \theta\right\} .
\end{aligned}
$$

By Lemma 3.1 this is equivalent to (3.38).

\section{HJBI-equations for stochastic differential games}

Problem A is related to the class of stochastic differential games studied in [MØ]. In the following we put $\mathcal{S}=(0, T) \times \mathbb{R}_{+}^{3}$ and $\tilde{\mathcal{S}}=(0, T) \times \mathbb{R}_{+}^{2}$.

By applying Theorem 3.2 in [MØ] to our situation we get the following verification theorem:

Theorem 4.1 [MØ]. (HJBI-equation)

Suppose $\varphi \in C^{1,2}(\mathcal{S}) \cap C(\overline{\mathcal{S}})$ and $(\hat{\theta}, \hat{\pi}) \in \mathbb{L} \times \Pi$ satisfy the following conditions:

(i) $A^{\theta, \hat{\pi}} \varphi(t, y)-\Lambda(\theta(t, \tilde{y})) \leq 0$ for all $\theta \in \Theta,(t, y) \in \mathcal{S}$

(ii) $A^{\hat{\theta}, \pi} \varphi(t, y)-\Lambda(\hat{\theta}(t, \tilde{y})) \geq 0$ for all $\pi \in \mathbb{R},(t, y) \in \mathcal{S}$

(iii) $A^{\hat{\theta}, \hat{\pi}} \varphi(t, y)-\Lambda(\hat{\theta}(t, \tilde{y}))=0$ for all $(t, y) \in \mathcal{S}$

(iv) $\varphi(T, k, s, x)=k g(s)-h(k, s)-k x$ for all $(k, s, x) \in \mathbb{R}_{3}^{+}$

(v) the family $\left\{\varphi\left(\tau, Y^{\theta, \pi}(\tau)\right)\right\}_{\tau \in \mathcal{T}}$ is uniformly integrable for all $(\theta, \pi) \in$ $\mathbb{L} \times \Pi, y \in \mathcal{S}$, where $\mathcal{T}$ is the set of all $\mathcal{F}_{t}$-stopping times $\tau \leq T$. 
Then

$$
\begin{aligned}
\varphi(t, y) & =\Phi_{G}(t, y)=\inf _{\pi \in \Pi}\left(\sup _{\theta \in \mathbb{L}} J^{\theta, \pi}(t, y)\right)=\sup _{\theta \in \mathbb{L}}\left(\inf _{\pi \in \Pi} J^{\theta, \pi}(t, y)\right) \\
& =\sup _{\theta \in \mathbb{L}} J^{\theta, \hat{\pi}}(t, y)=\inf _{\pi \in \Pi} J^{\hat{\theta}, \pi}(t, y)=J^{\hat{\theta}, \hat{\pi}}(t, y) ; \quad(t, y) \in \mathcal{S} .
\end{aligned}
$$

We can now state the first main theorem of this paper:

Theorem 4.2 Suppose the value function $\Psi_{G}(t, \tilde{y})$ for Problem $B$ satisfies the conditions of Lemma 3.2. Then the value function for Problem $A$ is

$$
\Phi_{G}(t, y)=\Psi_{G}(t, \tilde{y})-k x
$$

and there exists an optimal $\check{\theta} \in \mathbb{M}$ for Problem $B$ such that for all $\pi \in \Pi$ the pair

$$
\left(\theta^{*}, \pi^{*}\right)=(\check{\theta}, \pi)
$$

is an optimal pair for Problem A.

Proof. By the HJB equation for the stochastic control Problem B we know that

$$
\begin{aligned}
\sup _{\theta: M \theta}=0 & \left\{A^{\theta} \Psi_{G}(t, \tilde{y})-\Lambda(\theta(t, \tilde{y}))\right\} \\
& =A^{\check{\theta}(t, \tilde{y})} \Psi_{G}(t, \tilde{y})-\Lambda(\check{\theta}(t, \tilde{y}))=0, \quad \text { for } t \in(0, T),
\end{aligned}
$$

with terminal value

$$
\Psi_{G}(t, \tilde{y})=\Psi_{G}(t, k, s)=k g(s)-h(k, s) .
$$

Define

$$
\varphi(t, y)=\Psi_{G}(t, \tilde{y})-k x ; \quad(t, y) \in \mathcal{S}
$$

Then by Lemma 3.1 we have

$$
A^{\theta, \pi} \varphi(t, y)-\Lambda(\theta)=A^{\theta} \Psi_{G}(t, \tilde{y})-\Lambda(\theta)-k s \pi M \theta
$$

where $M \theta=M \theta(t, \tilde{y})$ is defined in (3.23). Therefore conditions (i)-(iii) of Theorem 4.1 get the form 
(i)' $A^{\theta} \Psi_{G}(t, k, s)-\Lambda(\theta)-k s \hat{\pi} M \theta(t, k, s) \leq 0$ for all $\theta \in \mathbb{R}^{2}$

(ii)' $A^{\hat{\theta}} \Psi_{G}(t, k, s)-\Lambda(\hat{\theta})-k s \pi M \hat{\theta}(t, k, s) \geq 0$ for all $\pi \in \mathbb{R}$

(iii)' $A^{\hat{\theta}} \Psi_{G}(t, k, s)-\Lambda(\hat{\theta})-k s \hat{\pi} M \hat{\theta}(t, k, s)=0$ for all $(t, k, s) \in \tilde{\mathcal{S}}$.

Choose $\hat{\pi}$ and $\check{\theta}=\hat{\theta}(\hat{\pi})$ as in Lemma 3.2. Combining (4.4) with Lemma 3.2 we get

$$
\begin{aligned}
& A^{\theta} \Psi_{G}-\Lambda \theta-k s \hat{\pi} M \theta \leq \sup _{\theta}\left\{A^{\theta} \Psi_{G}-\Lambda \theta-k s \hat{\pi} M \theta\right\} \\
& \quad=A^{\hat{\theta}(\hat{\pi})} \Psi_{G}-\Lambda \hat{\theta}(\hat{\pi})-k s \hat{\pi} M \hat{\theta}(\hat{\pi})=\sup _{\theta: M \theta=0}\left\{A^{\theta} \Psi_{G}-\Lambda \theta\right\}=0,
\end{aligned}
$$

which proves (i)'. Moreover, since $M \check{\theta}=0$ we get by (4.4)

$$
A^{\check{\theta}} \Psi_{G}-\Lambda \check{\theta}-k s \pi M \check{\theta}=A^{\check{\theta}} \Psi_{G}-\Lambda \check{\theta}=0 \quad \text { for all } \pi \in \mathbb{R},
$$

which proves (ii)' and (iii)'.

Finally we check that (iv) holds: By (4.5) and (4.4) we have

$$
\varphi(T, k, s, x)=\Psi_{G}(T, k, s)-k x=k g(s)-h(s, x)-k x .
$$

We conclude that $\varphi$ and $\hat{\theta}(\hat{\pi}), \hat{\pi}$ satisfy all the requirements of Theorem 4.1 and therefore

$$
\varphi(t, k, s, x)=\Phi_{G}(t, k, s, x)=\Psi_{G}(t, k, s)-k x .
$$

Moreover, $\theta^{*}:=\hat{\theta}(\hat{\pi})$ and $\pi^{*}:=\hat{\pi}$ constitute an optimal pair.

Now let $\pi \in \Pi$ be arbitrary. Note that

$$
E^{t, y}\left[K_{\theta^{*}}(T) X^{(\pi)}(T)\right]=E^{t, y}\left[K_{\check{\theta}}(T) X^{(\pi)}(T)\right]=k E_{\frac{1}{k} Q_{\check{\theta}}}^{t, y}\left[X^{(\pi)}(T)\right]=k x,
$$

since $\frac{1}{k} Q_{\check{\theta}}$ is an equivalent martingale measure. Therefore, going back to the definition of $\Phi_{G}$ we then have (see (3.26)-(3.28)), with $Y^{*}=Y^{\theta^{*}, \pi^{*}}, Y=Y^{\check{\theta}, \pi}$

$$
\begin{aligned}
& \Phi_{G}(t, y)=\inf _{\pi \in \Pi}\left(\sup _{\theta \in \mathbb{L}} J^{\theta, \pi}(t, y)\right)=J^{\hat{\theta}(\hat{\pi}), \hat{\pi}}(t, y) \\
& =E^{t, y}\left[-\int_{t}^{T} \Lambda\left(\hat{\theta}\left(t, \tilde{Y}^{*}(t)\right)\right) d t+K_{\theta^{*}}(T) g(S(T))\right.
\end{aligned}
$$




$$
\begin{aligned}
&\left.-h\left(K_{\theta^{*}}(T), S(T)\right)-K_{\theta^{*}}(T) X^{\left(\pi^{*}\right)}(T)\right] \\
&=E^{t, y}\left[-\int_{t}^{T} \Lambda\left(\hat{\theta}(t, \tilde{Y}(t)) d t+K_{\check{\theta}}(T) g(S(T))\right.\right. \\
&\left.-h\left(K_{\check{\theta}}(T), S(T)\right)\right]-k x=J^{\hat{\theta}(\hat{\pi}), \pi}(t, y) .
\end{aligned}
$$

We conclude that for all $\pi \in \Pi$ the pair

$$
\left(\theta^{*}, \pi\right)=(\check{\theta}, \pi) \in \mathbb{M} \times \Pi
$$

is optimal for Problem A, as claimed.

\section{$5 \quad$ Viscosity solutions}

In this section we present a viscosity solution approach to Theorem 4.2. The advantage with this approach is that it requires weaker assumptions on the value function.

The following definition is based on $[\mathrm{BI}]$ ( see also $[\mathrm{JK}]$ ).

\section{Definition 5.1 (Viscosity solutions)}

Let $\mathcal{C}$ denote the set of functions $u: \tilde{\mathcal{S}} \rightarrow \mathbb{R}$ at most linear growth

(i) An usc function $u \in \mathcal{C}$ is a viscosity subsolution of the HJB equation for Problem B, i.e.

$$
\begin{gathered}
\sup _{\theta: M \theta=0}\left\{A^{\theta} u-\Lambda(\theta)\right\}=0 \quad \text { in } \tilde{\mathcal{S}}, \\
u(T, \tilde{y})=k g(s)-h(\tilde{y})
\end{gathered}
$$

if $u$ satisfies (5.2) and for any $\varphi \in C^{2}\left(\mathbb{R}^{3}\right) \cap \mathcal{C}$ and $\left(t_{0}, \tilde{y}_{0}\right) \in \tilde{\mathcal{S}}$ such that $\varphi \geq u$ everywhere on $\tilde{\mathcal{S}}$ and $\varphi\left(t_{0}, \tilde{y}_{0}\right)=u\left(t_{0}, \tilde{y}_{0}\right)$ we have

$$
\sup _{\theta: M \theta=0}\left\{A^{\theta} \varphi-\Lambda(\theta)\right\}\left(t_{0}, \tilde{y}_{0}\right) \geq 0 .
$$

(ii) An lsc function $u \in \mathcal{C}$ is a viscosity supersolution of (5.1-5.2) if $u$ satisfies (5.2) and for any $\varphi \in C^{2}\left(\mathbb{R}^{3}\right) \cap \mathcal{C}$ and $\left(t_{0}, \tilde{y}_{0}\right) \in \tilde{\mathcal{S}}$ such that $\varphi \leq u$ everywhere on $\tilde{\mathcal{S}}$ and $\varphi\left(t_{0}, \tilde{y}_{0}\right)=u\left(t_{0}, \tilde{y}_{0}\right)$ we have

$$
\sup _{\theta: M \theta=0}\left\{A^{\theta} \varphi-\Lambda(\theta)\right\}\left(t_{0}, \tilde{y}_{0}\right) \leq 0 .
$$


(iii) A continuous function $u \in \mathcal{C}$ is a viscosity solution of (5.1-5.2) if $u$ is both a viscosity subsolution and a viscosity supersolution of (5.1-5.2).

A similar definition is given for a viscosity (sub/super) solution $u$ of the HJBI equation

$$
\begin{gathered}
\inf _{\pi \in \mathbb{R}}\left(\sup _{\theta \in \Theta}\left\{A^{\theta, \pi} u-\Lambda(\theta)\right\}\right)=0 \quad \text { in } \mathcal{S} \\
u(T, y)=k g(s)-h(\tilde{y})-k x
\end{gathered}
$$

for Problem A.

We say that a function $u \in C\left(\mathbb{R}^{3}\right) \cap \mathcal{C}$ satisfies the dynamic programming principle if

$$
u\left(t_{0}, \tilde{y}_{0}\right) \geq E^{t_{0}, \tilde{y}_{0}}\left[u\left(\tau, \tilde{Y}^{\theta}(\tau)\right)-\int_{0}^{\tau} \Lambda(\theta(s)) d s\right]
$$

for all bounded stopping times $\tau$ and all $\theta \in \Theta$ and all $\left(t_{0}, \tilde{y}_{0}\right) \in \mathbb{R}^{3}$.

Remark It is known that the dynamic programming principle holds under very general circumstances. See e.g. [I].

\section{Theorem 5.2}

(i) Suppose $u$ is a viscosity subsolution of the HJB equation (5.1-5.2) of Problem B. Then

$$
w(t, y):=u(t, \tilde{y})-k x
$$

is a viscosity subsolution of the HJBI equation (5.5-5.6) for Problem A.

(ii) Suppose $u$ satisfies (5.7) and (5.6). Then

$$
w(t, y):=u(t, \tilde{y})-k x
$$

is a viscosity supersolution of the HJBI equation (5.5-5.6) of Problem A.

(iii) Suppose $u$ satisfies (5.7) and $u$ is a viscosity subsolution of the HJB equation (5.1-5.2) of Problem B. Then

$$
w(t, y):=u(t, \tilde{y})-k x
$$

is a viscosity solution of the HJBI equation (5.5-5.6) of Problem A. 
Proof. It suffices to prove (i) and (ii).

Proof of (i): Suppose $u$ is a viscosity subsolution of (5.1). We want to prove that

$$
w(t, y):=u(t, \tilde{y})-k x
$$

is a viscosity subsolution of (5.5). To this end, suppose $\varphi \in C^{2} \cap \mathcal{C}, \varphi \geq w$ and $\varphi\left(t_{0}, y_{0}\right)=w\left(t_{0}, y_{0}\right)$ at some point $\left(t_{0}, y_{0}\right) \in \mathcal{S}$. Put

$$
\psi(t, y):=\varphi(t, y)+k x ; \quad(t, y) \in \mathcal{S} .
$$

Then

$$
\psi \in C^{2} \cap \mathcal{C}, \quad \psi \geq u \quad \text { and } \quad \psi\left(t_{0}, y_{0}\right)=u\left(t_{0}, y_{0}\right) .
$$

Therefore, since $u$ is a viscosity subsolution of (HJB) we have

$$
\sup _{\theta: M \theta=0}\left\{A^{\theta} \psi-\Lambda(\theta)\right\}\left(t_{0}, y_{0}\right) \geq 0 .
$$

But then, by Lemma 3.1,

$$
\begin{aligned}
& \inf _{\pi}\left(\sup _{\theta}\left\{A^{\theta, \pi} \varphi-\Lambda(\theta)\right\}\right)=\inf _{\pi}\left(\sup _{\theta}\left\{A^{\theta} \psi-\Lambda(\theta)+k_{0} s_{0} \pi M \theta\right\}\right) \\
& \quad \geq \inf _{\pi}\left(\sup _{\theta: M \theta=0}\left\{A^{\theta} \psi-\Lambda(\theta)+k_{0} s_{0} \pi M \theta\right\}\right)=\sup _{\theta: M \theta=0}\left\{A^{\theta} \psi-\Lambda(\theta)\right\} \geq 0 \text { at }\left(t_{0}, y_{0}\right) .
\end{aligned}
$$

This proves that $w$ is a subsolution of (HJBI).

Proof of part (ii): Suppose $u$ satisfies (5.7). We want to prove that

$$
w(t, y):=u(t, y)-k x
$$

is a viscosity supersolution of (5.5). To this end, let $\varphi \in C^{2} \cap \mathcal{C}, \varphi \leq w$ and $\varphi\left(t_{0}, y_{0}\right)=w\left(t_{0}, y_{0}\right)$. Define

$$
\psi(t, y)=\varphi(t, y)+k x .
$$

Then

$$
\psi \leq u \quad \text { and } \quad \psi\left(t_{0}, y_{0}\right)=u\left(t_{0}, y_{0}\right) .
$$

Therefore, since $u$ satisfies (5.7) we have

$$
\psi\left(t_{0}, \tilde{y}_{0}\right)=u\left(t_{0}, \tilde{y}_{0}\right) \geq E^{t_{0}, \tilde{y}_{0}}\left[u\left(\tilde{Y}^{\theta}(\tau)\right)-\int_{0}^{\tau} \Lambda(\theta) d s\right]
$$




$$
\geq E^{t_{0}, \tilde{y}_{0}}\left[\psi\left(\tilde{Y}^{\theta}(\tau)\right)-\int_{0}^{\tau} \Lambda(\theta) d s\right]
$$

By the Dynkin formula we have

$$
E^{t_{0}, \tilde{y}_{0}}\left[\psi\left(\tilde{Y}^{\theta}(\tau)\right)\right]=\psi\left(t_{0}, \tilde{y}_{0}\right)+E^{t_{0}, \tilde{y}_{0}}\left[\int_{0}^{\tau} A^{\theta} \psi\left(\tilde{Y}^{\theta}(s)\right) d s\right] .
$$

Combining (5.10) and (5.11) we get

$$
E^{t_{0}, \tilde{y}_{0}}\left[\int_{0}^{\tau}\left\{A^{\theta} \psi\left(\tilde{Y}^{\theta}(s)\right)-\Lambda(\theta(s))\right\} d s\right] \leq 0 .
$$

Since this holds for all bounded stopping times $\tau$ we conclude that

$$
A^{\theta} \psi-\Lambda(\theta) \leq 0 \quad \text { at } \quad\left(t_{0}, \tilde{y}_{0}\right) \forall \theta \in \Theta
$$

Hence

$$
\sup _{\theta}\left\{A^{\theta} \psi-\Lambda(\theta)\right\} \leq 0 \quad \text { at } \quad\left(t_{0}, \tilde{y}_{0}\right)
$$

Therefore

$$
\inf _{\pi}\left(\sup _{\theta}\left\{A^{\theta} \psi-\Lambda(\theta)-k_{0} s_{0} \pi M(\theta)\right\}\right) \leq 0 \quad \text { at } \quad\left(t_{0}, \tilde{y}_{0}\right) .
$$

This proves that $w$ is a supersolution of (HJBI), and hence completes the proof of (ii).

Using Theorem 5.2 we can now state the following viscosity solution version of Theorem 4.2:

Theorem 5.3 As before let $\Phi_{G}(t, y)=\Phi_{G}(t, k, s, x)$ and $\Psi_{G}(t, \tilde{y})=\Psi_{G}(t, k, s)$ be the value functions of Problem $A$ and Problem $B$, respectively. Suppose that $\Phi_{G}(t, k, s, x)$ is the unique viscosity solution of the HJBI equation (5.55.6) for Problem A. Then

$$
\Phi_{G}(t, k, s, x)=\Psi_{G}(t, k, s)-k x .
$$


Proof. By $[\mathrm{P}]$, Theorem 3.1 we know that $\Psi_{G}(t, k, s)$ is a viscosity solution of the HJB equation (5.1) for Problem B. Moreover, $\Psi_{G}(t, k, s)$ satisfies the dynamic programming principle (5.7). Hence by Theorem 5.2 we get that

$$
u(t, k, s, x):=\Psi_{G}(t, k, s)-k x
$$

is a viscosity solution of the HJBI equation (5.5- 5.6) for Problem A. Therefore, by uniqueness

$$
\Phi_{G}(t, k, s, x)=u(t, k, s, x)=\Psi_{G}(t, k, s)-k x .
$$

Remark 5.4 Sufficient conditions for the uniqueness of the viscosity solutions of the HJBI equation (5.5) are given in $[\mathrm{JK}]$ and $[\mathrm{P}]$. For example, it suffices to assume that the jumps sizes

$$
|\Delta \eta(t)|=\left|\eta(t)-\eta\left(t^{-}\right)\right|
$$

are bounded away from 0 . See $[\mathrm{P}]$, Theorem 4.1, whose proof seems to require that such a condition holds.

\section{Risk indifference pricing}

We now apply Theorem 4.2 or Theorem 5.3 to find the risk indifference price $p=p_{\text {risk }}$ given in Definition 2.1, i.e. given as the solution $p$ of the equation

$$
\Phi_{G}(t, k, s, x+p)=\Phi_{0}(t, k, s, x)
$$

where $\Phi_{G}$ is the solution of Problem A. By Theorem 4.2 or Theorem 5.3 this equation becomes

$$
\Psi_{G}(t, k, s)-k(x+p)=\Psi_{0}(t, k, s)-k x
$$

which has the solution

$$
p=p_{\text {risk }}=k^{-1}\left(\Psi_{G}(t, k, s)-\Psi_{0}(t, k, s)\right) .
$$

In particular, choosing $k=1$ (i.e. all measures $Q \in \mathcal{L}$ are probability measures), we get 
Theorem 6.1 (Risk indifference pricing theorem - seller's price) Suppose that either the conditions of Theorem 4.2 or the conditions of Theorem 5.3 hold. Then the seller's risk indifference price of $G, p_{\text {risk }}^{\text {seller }}(G)$, is given by

$$
p_{\text {risk }}^{\text {seller }}(G)=\sup _{Q \in \mathcal{M}}\left\{E_{Q}[G]-\zeta(Q)\right\}-\sup _{Q \in \mathcal{M}}\{-\zeta(Q)\},
$$

where $\mathcal{M}$ is the set of equivalent martingale measures defined by (3.21).

Remark 6.2 Note that

$$
\begin{aligned}
p_{\text {risk }}^{\text {seller }}(G) & \leq \sup _{Q \in \mathcal{M}} E_{Q}[G]+\sup _{Q \in \mathcal{M}}\{-\zeta(Q)\}-\sup _{Q \in \mathcal{M}}\{-\zeta(Q)\} \leq \sup _{Q \in \mathcal{M}_{1}} E_{Q}[G] \\
& =p_{\text {up }}(G), \quad \text { the upper hedging price of } G \text { (see }(2.1) \text { ), }
\end{aligned}
$$

with equality only if $\zeta(Q)=0$ for all $Q$.

Similarly we get

Theorem 6.3 (Risk indifference pricing theorem - buyer's price) Suppose that either the conditions of Theorem 4.2 or the conditions of Theorem 5.3 hold. Then the buyer's risk indifference price of $G, p_{\text {risk }}^{\text {buyer }}(G)$, is given by

$$
p_{\text {risk }}^{\text {buyer }}(G)=\inf _{Q \in \mathcal{M}}\left\{E_{Q}[G]+\zeta(Q)\right\}-\inf _{Q \in \mathcal{M}} \zeta(Q) .
$$

Remark 6.4 Note that

$$
p_{\text {risk }}^{\text {buyer }}(G) \geq \inf _{Q \in \mathcal{M}} E_{Q}[G]=p_{\text {low }}(G),
$$

the lower hedging price of $G$.

Combining this with (6.4) we get the following chain of inequalities:

\section{Corollary 6.5}

$$
p_{\text {low }}(G) \leq p_{\text {risk }}^{\text {buyer }}(G) \leq p_{\text {risk }}^{\text {seller }}(G) \leq p_{\text {up }}(G) .
$$


Proof. It remains to prove the second inequality, namely that

$$
\begin{aligned}
\inf _{Q \in \mathcal{M}}\left\{E_{Q}[G]+\zeta(Q)\right\}-\inf _{Q \in \mathcal{M}} \zeta(Q) \\
\leq \sup _{Q \in \mathcal{M}}\left\{E_{Q}[G]-\zeta(Q)\right\}+\inf _{Q \in \mathcal{M}} \zeta(Q) .
\end{aligned}
$$

Now

$$
\begin{aligned}
& \sup _{Q \in \mathcal{M}}\left\{E_{Q}[G]-\zeta(Q)\right\}-\inf _{Q \in \mathcal{M}}\left\{E_{Q}[G]+\zeta(Q)\right\} \\
& \geq \sup _{Q \in \mathcal{M}}\left\{E_{Q}[G]-\zeta(Q)-\left(E_{Q}[G]+\zeta(Q)\right)\right\} \\
& =\sup _{Q \in \mathcal{M}}\{-2 \zeta(Q)\}=-2 \inf _{Q \in \mathcal{M}} \zeta(Q),
\end{aligned}
$$

from which (6.8) follows.

From (6.9) we deduce the following:

Corollary 6.6 Suppose

$$
\underset{Q \in \mathcal{M}}{\operatorname{Argmax}}\left\{E_{Q}[G]-\zeta(Q)\right\} \cap \underset{Q \in \mathcal{M}}{\operatorname{Argmin}}\left\{E_{Q}[G]+\zeta(Q)\right\} \neq \emptyset .
$$

Then

$$
p_{\text {risk }}^{\text {buyer }}(G)=p_{\text {risk }}^{\text {seller }}(G) .
$$

Note that (6.10) holds trivially if $\mathcal{M}$ consists of just one measure, which corresponds to the case when the market is complete.

\section{Concluding remarks}

In general the gap $p_{\text {up }}(G)-p_{\text {low }}(G)$ between the upper and lower hedging prices is too wide to make either of them a good candidate for the trading price in an incomplete market. Our result (6.7) shows that by using the risk indifference pricing principle, the gap between the seller's and the buyer's price gets smaller.

Acknowledgments. We are grateful to Yeliz Yolcu Okur and Martin Schweizer for helpful comments. 


\section{References}

[ADEH] P. Artzner, F. Delbaen, J. M. Eber and D. Heath: Coherent measures of risk. Mathematical Finance 4 (1999), 203-228.

[Ap] D. Applebaum: Lévy Processes and Stochastic Calculus. Cambridge Univ. Press 2003.

[BE] P. Barrieu and N. EL Karoui: Optimal derivatives design under dynamic risk measures. Mathematics of Finance, Contemporary Mathematics (A.M.S. Proceedings), 13-26, 2004.

[B] J. Bertoin: Lévy Processes. Cambridge Univ. Press 1996.

[BeM] F. E. Benth and T. Meyer-Brandis: The density process of the minimal entropy martingale measure in a stochastic volatility model with jumps. Finance and Stochastics, Vol. 9(4), 563-575, 2005.

[BI] G. Barles and C. Imbert: Second-order elliptic integro-differential equations: Viscosity solutions' theory revisited, Annales de l'IHP, Vol. 25(3) (2008), 567-585.

[D] F. Delbaen: Coherent risk measures on general probability spaces. In K. Sandmann and J. Schonbucher (eds.): Advances in Finance and Stochastics, Essays in Honor of Dieter Sondermann. Springer 2002, 1-37.

[FR] M. Frittelli and E. Rosazza-Gianin: Putting order in risk measures. J. Banking and Finance 26 (2002), 1473-1486.

[FS] H. Föllmer and A. Schied: Convex measures of risk and trading constraints. Finance Stochast. 2 (2002), 429-447.

[HN] S. D. Hodges and A. Neuberger: Optimal replication of contingent claims under transaction costs. Rev. Future Markets 8 (1989), 222-239.

[I] Y. Ishikawa: Optimal control problem associated with jump processes. Appl. Math. Opt. 50 (2004), 21-65.

[JK] E. R. Jakobsen and K. H. Karlsen: A maximum principle for semicontinuous functions applicable to integro-partial differential equations. Nonlinear Diff. Equ. Appl. 13 (2006), 1-29. 
[Ku] H. Kunita: Representation of martingales with jumps and application to mathematical finance. Advanced Studies in Pure Mathematics 41 (2004). Stochastic Analysis and Related Topics, 209-232.

[KS] S. Klöppel and M. Schweizer: Dynamic utility indifference valuation via convex risk measures. NCCR FINRISK Working Paper No 209, 2005.

[MØ] S. Mataramvura and B. Øksendal: Risk minimizing portfolios and HJB equations for stochastic differential games. Stochastics, Vol 80(4) (2008), $317-337$.

[MZ] M. Musiela and T. Zariphopolou: An example of indifference prices under exponential preferences. Finance Stochast. 8 (2004), 229 - 239.

[ØS] B. Øksendal and A. Sulem: Applied Stochastic Control of Jump Diffusions. Second Edition. Springer 2007.

[ØS2] B. Øksendal and A. Sulem: A game theoretic approach to martingale measures in incomplete markets. Surveys of Applied and Industrial Mathematics, 15 (2008), 18 - 24.

[P] H. Pham: Optimal stopping of controlled jump diffusion processes: A viscosity solution approach. J. Math. Syst. Est. Control 8 (1998), 1-27.

[Sa] K. Sato: Lévy Processes and Infinitely Divisible Distributions. Cambridge Univ. Press 1999.

[ST] A. Sulem and P. Tankov: Utility-based pricing and hedging in models with jumps. Manuscript 2006.

[X] M. Xu: Risk measure pricing and hedging in incomplete markets. Annals of Finance 2 (2006), 51-71. 